\title{
Motivational Factors in Educational MMORPGs: Some Implications for Education
}

\author{
Kuo-Hsun Hung, Charles Kinzer, and Cheng-Ling Alice Chen \\ Box 8, 525 W 120th Street, New York, New York 10027-6696 \\ Location: 322 Thompson Hall \\ kh2203@columbia.edu, kinzer@tc.columbia.edu, cc2550@columbia.edu
}

During the past two decades, digital games have been acknowledged as motivational and have become a source of study for educational researchers and instructional designers who wish to maximize motivation in educational materials. Among different types of digital games, massively multiplayer online role-playing games (MMORPGs) continue to grow in popularity. Therefore, researchers have started to explore the possibilities of using MMORPGs in education. While MMORPGs have been proven to be a tool that can strengthen students' learning, to enhance their efforts educational game designers need to know more about how educational MMORPGs motivate students. To address the above need, we set out the following two objectives for the study briefly reported herein: To examine the motivational factors of playing educational MMORPGs; To discuss how educators and educational game designers could use motivational factors in designing learning activities and educational experiences within MMORPGs.

We used a self-developed educational MMORPG called Ed-Wonderland to explore the two objectives noted earlier. Twenty $5^{\text {th }}$ grade students $($ female $=10$, male $=10$ ) school in KaoHsiung, Taiwan agreed to participate. We first conducted a twentyminute session to introduce Ed-Wonderland and its game world, rules, and functions. This was followed by a one-hour play session. After the play session, each participant completed a motivation questionnaire and overall attitude questionnaire.

The results show that participants are motivated by Achievement, Social, Immersion, and Instructional Mechanism Factors. In addition, participants' attitudes toward Ed-Wonderland are positive.

These findings point to the following implications for both educators and educational game designer. For educators, we suggest that (1) educational MMORPGs such as Ed-Wonderland could be used to motivate students to learn; (2) educational MMORPGs such as Ed-Wonderland could be a social learning platform for after class usage; and (3) educators could utilize the four motivational factors in their learning activities. The implications for educator games designers are (1) the instructional materials and tools should be thorough; and (2) educational games could be accompanied with other materials. 